\title{
The survey for new AGN candidates within the field of Fermi unassociated gamma-ray sources
}

\author{
Yoshitaka Fujinaga*, Kotaro Niinuma, Atushi Kimura, Kenta Fujisawa, Kengo \\ Akutagawa, Kazuhito Motogi \\ Yamaguchi University \\ E-mail: t017vc@yamaquchi-u.ac.jp
}

Koichiro Sugiyama

Ibaraki University

\section{Tomoaki Oyama, Yusuke Kono, Satoko Sawada-Sato}

National Astronomical Observatory of Japan

\section{Syota Mizuno}

Advanced Engineering Service

\section{Yoshihiro Fukuzaki}

The Geospatial Information Authority of Japan

\section{Shinji Takemura}

Gifu University

\begin{abstract}
We report on the results of survey for new $\gamma$-ray AGN candidates by using Japanese VLBI Network. Aims of the survey are 1) verification of Blazar Sequence with taking account of faint blazars and 2) discovery of new types of AGN with $\gamma$-ray emission. To achieve our purpose, we observed all NVSS and FIRST sources located within the positional uncertainties of unassociated $\gamma$-ray sources listed in Fermi/LAT 2nd catalog. As a result of our JVN observation of 845 radio sources, we detected 29 VLBI sources. In our poster, we will show not only the results of estimations of types of these sources but also several correlation diagrams of them.
\end{abstract}

12th European VLBI Network Symposium and Users Meeting

7-10 October 2014

Cagliari, Italy

\footnotetext{
* Speaker.
} 


\section{Introduction}

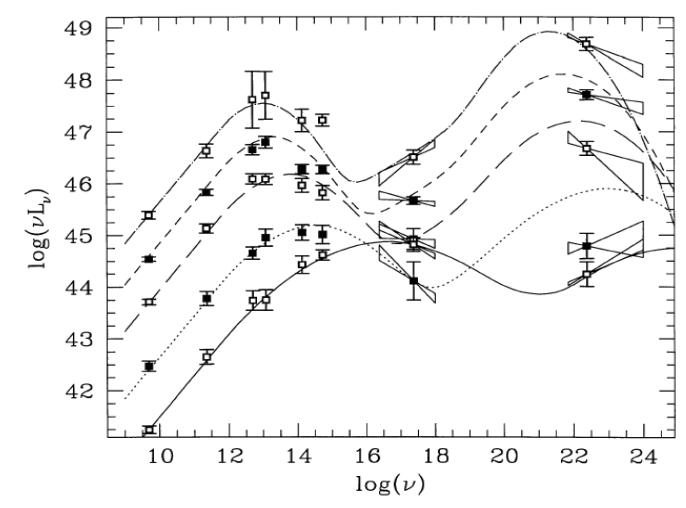

Figure 1: SED of Blazar (Fossati et al. 1998)

Blazars are sub-class of AGNs with relativistic jets pointin almost along the line of sight. Blazar's Spectrum Energy Distributions (SEDs) have two humps (Figure 1). Left humps are emitted by synchrotron emission and right humps are emitted by inverse-Compton scattering. Peak frequency and peak luminosity show anti-correlation in synchrotron components. This characteristic is called Blazar Sequence (Fossati et al. 1998). However, this characteristic was discovered among bright blazars at radio and it is needed to verify "Blazar Sequence" with taking account of faint blazars at radio. Therefore, We conducted Blazar survey by high sensitive VLBI to increase the number of faint radio samples and to verify Blazar Sequence.

\section{Sample selection and Observation}

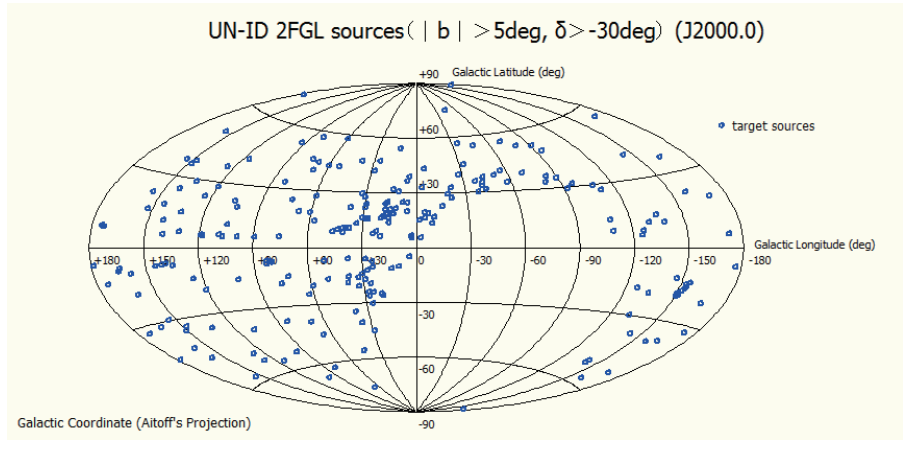

Figure 2: The distribution of selected $\gamma$-ray sources at galactic coordinate.

Our observation samples were all radio sources located within the positional uncertainties of unassociated $\gamma$-ray sources listed in Fermi 2nd Catalog (Nolan et al. 2012). $1873 \gamma$-ray sources are listed in Fermi 2nd Catalog and $1298 \gamma$-ray sources in Fermi 2nd Catalog are associated with the sources at other bandwidth. Because blazar are the majority of $\gamma$-ray sources (62\% out of 1298 $\gamma$-ray sources), we can expect that unassociated $\gamma$-ray sources contain many blazars. To select possible extragalactic and observable source, we applied the following two criteria to unassociated 
$\gamma$-ray sources: (1) Galactic latitude $|b|>5^{\circ}$. (2) Declination $\delta>-30^{\circ}$. We selected $231 \gamma$-ray sources from 575 unassociated $\gamma$-ray sources by these criteria. Next, we searched for radio sources located within the positional errors of $232 \gamma$-ray sources from the NVSS (Condon et al. 1998) catalog and FIRST (Becker et al. 1995) catalog. We obtained 1211 candidate radio sources in this process.

We conducted an optical-fiber-linked real-time VLBI observations, which is constructed by Optically ConnecTed Array for VLBI Exploration project (OCTAVE) operated as a sub-array of JVN for the total of 845 radio sample sources (located within the positional error of $150 \gamma$-ray sources). Observations were performed in five sessions (Table 1) using only one baseline composed of Yamaguchi $32 \mathrm{~m}$ and Tsukuba $32 \mathrm{~m}$ telescope, which has baseline length of $804 \mathrm{~km}$, at $8.4 \mathrm{GHz}$ with a bandwidth of $512 \mathrm{MHz}(8192-8704 \mathrm{MHz})$. Typical angular resolution and expected $1 \sigma$ noise level are 9.2 mas and $0.83 \mathrm{mJy}$ with an on-source time of three minutes, respectively.

Table 1: Informations of five observation sessions.

\begin{tabular}{|c|c|c|}
\hline Observation code & Time (UT) [DOY hh:mm:ss] & Observed counts \\
\hline \hline S12335A & $33600: 43: 45-33606: 56: 30$ & 85 \\
\hline S12336A & $33609: 30: 00-33706: 56: 50$ & 248 \\
\hline S12337B & 337 18:15:00 - 337 23:23:20 & 66 \\
\hline S12342A & $34218: 30: 00-34306: 59: 25$ & 154 \\
\hline S12359A & $35901: 00: 00-35923: 27: 15$ & 292 \\
\hline
\end{tabular}

\section{Result and Discussion}

As a results of five sessions, we detected 29 radio sources. We found that 27 out of $150 \gamma$-ray sources have one radio counterpart and one $\gamma$-ray source has two radio counterparts. In the point of view of brightness temperature, we estimated that detected sources were AGNs (active galactic nuclei) candidates because all detected sources have higher brightness temperatures than $10^{6} \mathrm{~K}$. We could not obtain the structures of sources and spectral indices because our observations are conducted at one band by one baseline. Therefore, we could not estimate the class of detected sources only our observations.

The angular separation between the NVSS or FIRST and the VLBI positions is expected to be smaller than 1".5 because typical positional error of NVSS and FIRST catalogs range from 0". 5 to $1 " .5$ depending on the flux density. Therefore, based on the radio coordinates, we could search for multi-wavelength counterparts to newly detected VLBI sources effectively. In this procedure, we used the ninth data release of the Sloan Digital Sky Survey (SDSS; Ahn et al. 2012) catalog, the Two Micron All Sky Survey (2MASS; Sukrutskie et al. 2006) catalog and the WISE All-Sky Source Catalog (WISE; Wright et al. 2010).

To identify the classes of these AGN candidates, we applied the method proposed by Massaro et al. (2012) to these sources. Based on Massaro et al. (2012), they defined the narrow subregion delineated in WISE color-color diagrams using magnitude at $3.4 \mathrm{~m}, 4.6 \mathrm{~m}, 12 \mathrm{~m}$ and $22 \mathrm{~m}$ (hereafter [3.4], [4.6], [12] and [22], respectively) as WGS in which $\gamma$-ray blazars are located and introduced the parameter s that identify whether the sources were included in WGS. To utilize this method, we need magnitude and error information at each band of WISE data. We cannot apply this method to the sources, if any band shows upper limit. Although 22 out of 29 detected or marginally 
detected sources have WISE candidates, the number of the source given the magnitude and error informations at each band is only 14 . As a result of applying this method, we classified seven $\gamma$-ray sources into BL Lacs and two $\gamma$-ray sources into FSRQ.

We conducted multi-band following observation at S, X and U band by VLBA (Very Long Baseline Array) for 6 out of 29 detected sources. We show the results of NVSS J030727+491510 as one example (Figure 3). The spectral index of this source calculated from the results of multiband observation was $0.07\left(S \propto v^{-\alpha}\right)$, which suggest that this source is possible blazar because the spectrum at $2-15 \mathrm{GHz}$ is flatter than the typical spectral index of blazar $(\sim 0.3)$. As a results of estimation of spectral indices of other five sources in the same way, we found that six out of five sources were possible blazar.

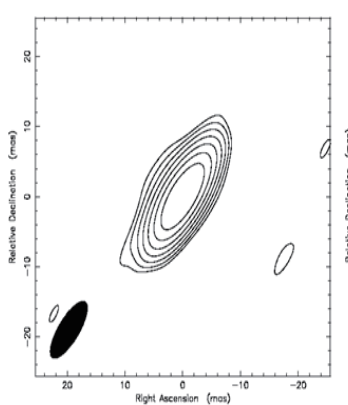

Peak flux: 0.272[Jy/beam] Beam size: $9.7 \times 3.4[\mathrm{mas}]$
Image rms: $2[\mathrm{mJy}]$

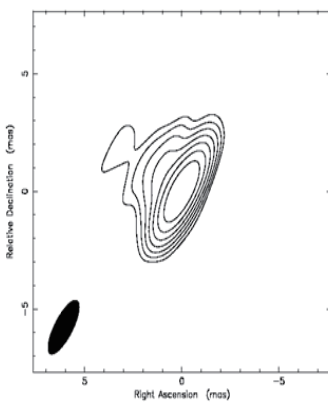

Peak flux:0.249[Jy/beam] Beam size: $2.6 \times 0.9$ [mas] Image rms: 2 [mJy]

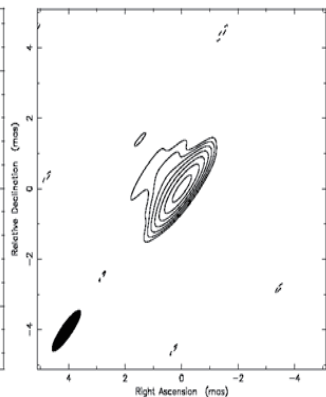

Peak flux:0.204[Jy/beam] Beam size: $1.5 \times 0.37$ [mas] Image rms:2 [mJy]

Figure 3: VLBA images of NVSS J030727+491510 at $2.3 \mathrm{GHz}$ (left), $8.4 \mathrm{GHz}$ (center), and $15 \mathrm{GHz}$ (right).

Also, we compared the correlation diagram of detected sources with the one of 2LAC (Ackermann et al. 2011). Trend of correlation between photon flux at $\mathrm{GeV}$ band $\mathrm{F}_{1-100 \mathrm{GeV}}$ and flux density at radio $S_{1.4 \mathrm{GHz}}\left(\log \mathrm{F}_{1-100 \mathrm{GeV}}-\log \mathrm{S}_{1.4 \mathrm{GHz}}\right)$ show that detected sources are possibly HSP (high synchrotron peaked) BL Lacs. On the other hand, that between $F_{1-100 \mathrm{GeV}}$ and photon index at $\mathrm{GeV}$ band $\Gamma_{1-100 \mathrm{GeV}}\left(\log \mathrm{F}_{1-100 \mathrm{GeV}}-\Gamma_{1-100 \mathrm{GeV}}\right)$ show detected sources are possibly FSRQs (Flat Spectrum Radio Quasars). These trends possibly show that detected sources are different trend from 2LAC blazars.

\section{References}

[1] Fossati, G., Maraschi, L., Celotti, A., \& Ghisellini, G. 1998, MNRAS, 299, 433

[2] Nolan, P.L., et al. 2012, ApJS, 199, 31

[3] Condon, J. J., et al. 1998, AJ, 115, 1693

[4] Becker, R. H., White, R. L. \& Helfand, D. J. 1995, ApJ, 450, 559

[5] Ahn, C. P., et al. 2012, ApJS, 203, 21

[6] Skrutskie, M. F., et al. 2006, ApJ, 131, 1163

[7] Wright, E. L., et al. 2010, ApJ, 140, 1868

[8] Massaro, F., et al. 2012, ApJ, 750, 138

[9] Ackermann, M., et al. 2011, ApJ, 743, 171 